\title{
Erratum to: On Replica Placement in High-Availability Storage Under Correlated Failure
}

\author{
K. Alex Mills ${ }^{(\bowtie)}$, R. Chandrasekaran, and Neeraj Mittal \\ Department of Computer Science, The University of Texas at Dallas, \\ Richardson, TX, USA \\ \{k.alex.mills, chandra, neerajm\}@utdallas.edu
}

\section{Erratum to: \\ Chapter 26: Z. Lu et al. (Eds.) \\ Combinatorial Optimization and Applications \\ DOI: $10.1007 / 978-3-319-26626-8 \_26$}

On page 351 of the original version of the paper, there was a typo in the formula defining the lexicographic order. Because the failure aggregate was indexed using decreasing indices, the direction of one of the inequalities should be reversed. The corrected formula is stated below:

$$
\boldsymbol{f}(P) \leq_{L} \boldsymbol{f}\left(P^{\prime}\right) \Longleftrightarrow \exists m>0,\left[\forall i>m: p_{i}=p_{i}^{\prime}\right] \wedge p_{m} \leq p_{m}^{\prime} .
$$

In the proof of Theorem 1 on page 354, the last sentence of paragraph 3 was modified to read as follows: "Let $S^{-}$(resp. $S^{+}$) be the set of nodes whose failure numbers change from $\alpha$ (resp. change to $\alpha$ ), as a result of the swap." The modified words have been italicized for emphasis. This phrasing is consistent with the formal definitions of $S^{+}$and $S^{-}$given immediately below, which are correct as stated in the original paper.

At the top of page 356, the function Label-Children should take as arguments the set $\left\{c_{1}, c_{2}, \ldots, c_{k}\right\}$, and a number $r$. The parentheses were suppressed during editing.

Starting from the statement of Theorem 2 on page 356, terms such as $k, \frac{|U|}{k}, \frac{|U|-1}{k}$, and $|U| \bmod k$ begin to appear in several formulas. With the usual meaning of $|U|$ and $k$ the formulas do not read correctly. In the corrected paper, we have introduced a new term, $R$, which denotes the number of replicas to be distributed among the unfilled children. If $F$ and $U$ are the sets of filled and unfilled children of node $u$ found by Algorithm 1, then $R$ is formally defined as $R=r(u)-\sum_{c_{i} \in F} \ell_{i}$. In each of the terms mentioned, replace each instance of $|U|$ by $R$ and each instance of $k$ by $|U|$. With these changes, the paper reads correctly. 